\title{
L'intraduisible et l'implicite. Frontières ou zones de contact en traduction ?
}

\author{
Merete Birkelund et Sébastien Doubinsky (Université d'Aarhus, \\ Danemark)
}

\begin{abstract}
The untranslatable and the implicit often represent the two limits of translation - the untranslatable by what can be expressed in only one language, and the implicit by what is not expressed in one language. Phenomena in a constant margin, phenomena in a liminary position (see Jacques Derrida), they overlap several territories, ranging from cultural to linguistic. Between the "nothing is translatable" and the "everything is translatable" delimited by Susan Apter, and the possible or impossible equivalences of Eugene Nida, these two notions are always troublesome, or at least, in motion. Indeed, the definitions themselves are sometimes blurred. What is untranslatable? Does "intraductible" mean inexplicable, unpredictable or incomprehensible? And how can we determine the implicit, since it is, above all, an absence, a meaning that lies beyond or below the text? However, despite this apparent absence, the transfer of the unsaid can be translated, but often by other linguistic means than those used in the source language.

Since languages (in this context French and Danish) are not isomorphic, translation remains intimately linked to this reality. The task of the translator is to know how to transmit the combination of the implicit and the explicit, but also to try to explain what at first sight seems untranslatable in order to respect the (implicit) intention of the discourse of the language source.
\end{abstract}

Keywords: translation, the untranslatable, the implicit, transfer of the unsaid, irony

\section{Introduction}

L'intraduisible et l'implicite peuvent être considérés comme la limite extrême de la traduction. Bien que les deux phénomènes soient intrinsèquement liés à toute communication linguistique, que ce soit la communication unilingue ou la rencontre entre deux langues, ils représentent un défi dans l'activité traduisante. En effet, ce sont deux notions qui impliquent soit un échec - ce qui ne peut pas être traduit -, soit la possibilité d'un échec - ce qui risque de ne pas pouvoir être traduit. Ce sont pourtant deux notions incontournables dans le champ de la traduction, et auxquelles il faut s'attaquer si l'on veut pouvoir avoir une vision complète des problèmes et des problématiques qui y sont attachés. 


\section{L'intraduisible et ses sous-champs}

Si ce qu'on appelle l'intraduisible semble, de prime abord, évident, il pose néanmoins de sérieux problèmes de définition, qu'il nous faut examiner pour mieux en définir la nature, ses limites, voire ses possibilités.

Le Larousse en ligne, pourtant, choisit le camp apparent de la simplicité. Voici les deux définitions qu'il en donne:

1. Qu'il est impossible de traduire dans une autre langue : Texte intraduisible.

2. Qu'il est impossible d'exprimer : Un sentiment intraduisible.

http://www.larousse.fr/dictionnaires/francais/intraduisible/43958\#DUzgQ7zRQbQS0xG c. 99

Or si ces définitions sont exactes, elles sont tout sauf précises. En effet, 'intraduisible' recouvre une série de sous-notions qui délimite chacune un aspect différent du problème. $\mathrm{Ce}$ sont des sous-champs particuliers qui impliquent chacun une approche spécifique ainsi qu'une position originale dans le champ de 'l'intraduction'.

Nous pouvons les catégoriser ainsi :

1. L'intraduisible : ce qui ne peut pas être traduit car n'existant pas comme expression, ou comme référence, dans la langue-cible. Concerne essentiellement le domaine linguistique, et les difficultés de correspondance absolue entre langues. C'est le terrain des 'équivalences' d'Eugène Nida (1964), où l'impossible 'correspondance absolue' entre deux langues est résolue au moyen de mots ou d'expressions sémantiquement semblables.

2. L'intraductible: ce qui ne peut pas être transmis et/ou compris dans une autre langue. Il s'agit ici de références culturelles typiques ou locales, qui n'appartiennent qu'à la langue et à la culture-source. Nous touchons ici au domaine des linguistes, Edward Sapir et Lee Whorf (1921), pour qui les mots et les expressions n'auront jamais le sens, même s'ils peuvent sembler équivalents, du fait du cadre culturel idiosyncratique dans lequel ils sont produits.

3. L'intraduit : ce qui intègre la langue-cible en gardant plus ou moins sa morphologie d'origine. Ce sont les fameux 'emprunts' que l'on trouve parsemés dans notre langue, de « goulash» à « weekend».

Ces aspects ou zones représentent chacun une situation particulière pour le traducteur, qui apparaissent bien souvent comme les frontières de sa propre langue. Et si, comme nous l'avons signalé plus haut, chacun de ces aspects renvoie à une situation ou une position particulière, ils infèrent aussi, de la part dudit traducteur, une recherche de solutions différentes.

\subsection{L'intraduisible}

L'intraduisible, par exemple, sera le plus souvent contourné par le système d'équivalences proposé par Eugène Nida (1964), qui suggère une substitution sémantique trouvant son origine dans la langue cible, et 'correspondant' à l'expression de la langue source. Un exemple connu : «Raining cats and dogs » en anglais qui devient «pleuvoir des 
cordes» ou «pleuvoir des hallebardes» en français. Il est évident ici qu'une traduction littérale n'aurait aucun sens en français, et qu'une adaptation à partir d'une notion équivalente est nécessaire. L'intraduisible est la marque de la différence culturelle de la langue source au travers de son vocabulaire, qui peut cependant être contournée par la souplesse et la richesse du corpus de la langue cible. C'est la première ligne de difficulté de la traduction, mais celle aussi qui semble pouvoir être la plus facilement contournable.

\subsection{L'intraductible}

De même, l'intraductible, qui a été abordé par Edward Sapir et Lee Whorf (1921), concerne les éléments de langage qui sont si intimement liés à la culture-source, qu'ils sont impossibles à traduire dans la langue cible. Comme exemple, on pourrait prendre le terme japonais «Yugen » qui signifie, selon la Wikipédia anglo-saxonne : «a profound, mysterious sense of the beauty of the universe ... and the sad beauty of human suffering » (https://en.wikipedia.org/wiki/Japanese_aesthetics\#Y\%C5\%ABgen) ('Un sens mystérieux et profond de la beauté de l'univers et de la souffrance humaine'. (notre traduction)). Si l'on peut 'comprendre' le concept, comme mot, il est directement intraduisible - du moins en français. (Le sehnsucht allemand pourrait par contre s'en rapprocher - mais ce mot est aussi directement 'intraductible' en français...). Il s'agit donc ici de mots dont la langue cible n'a aucun équivalent direct, ni aucune 'équivalence' évidente.

Bien évidemment se pose ici la fracture importante de 'ce que l'on peut' ou 'ne peut pas' comprendre, qui va déterminer le sens même du mot 'traduction'. Si 'traduire' veut simplement dire 'rendre compréhensible', alors nous n'avons plus de problème et le modèle des équivalences de Nida devrait, seul, fonctionner sans problème. Après tout, comme il l'a expliqué lui-même dans Principles of correspondance, aucune langue ne correspondant parfaitement, il est impossible d'établir une égalité de sens parfaite (Nida, in Venuti, 2004 : 153). Cependant, ce que nous nommons 'compréhensible' risque alors de devenir une simple 'équivalence' de pensée, et non la restitution d'une pensée propre. C'est le sens de la critique d'Antoine Berman (1984) et de Lawrence Venuti (1998), qui considéreront cette forme de traduction comme une domestication de la langue et, à travers elle, de la culture du textesource. Le travail d'Eric Auerbach (1946) sur la notion de la Mimêsis grecque nous fait ainsi bien voir l'évolution, non pas du mot, mais du sens du mot à travers l'histoire. Si nous comprenons le mot aujourd'hui, nous ne le comprenons pas de la même manière qu'Aristote au IVème siècle avant Jésus-Christ, ou qu'à la Renaissance. Le sens a évolué, s'est enrichi ou appauvri (c'est selon), et s'est déformé. Surtout, il a été 'approprié' au cours des siècles par des cultures différentes, chacune avec son enjeu, ses désirs et son intention propres.

À l'inverse, pour revenir à «Yugen», il est évident que ce terme exprime implicitement tout une pensée esthétique et philosophique qui, a priori, nous échappe complètement. Le réseau sémantique et sémiotique auquel il appartient correspond à la fameuse 'Rhétorique' chère à Gayatri Spivak (1993/2004), qui recouvre tout ce qui n'est justement pas contenu dans le mot ou l'expression - cet 'implicite' qui va être traité plus loin dans la section 2 ci-dessus) mais qu'il est nécessaire - ou 'obligatoire' comme le dit Spivak, de connaître pour pouvoir offrir une traduction correcte. C'est plus qu'un mot, c'est toute une culture. 
Une des possibilités de résolution de ce problème nous est offerte par Lawrence Venuti (1995) et sa méthode dite de foreignization du texte-cible, c'est-à-dire de se cantonner au plus près de la littéralité originelle du texte-source, quitte à 'maltraiter' la langue cible. Le lecteur devra ainsi faire un effort pour lire la traduction, qui lui indiquera, par sa forme parfois difficile, en tout cas particulière, qu'il faut chercher plus loin le sens de ce qu'il lit, dans la culture d'où vient l'ouvrage. C'est 'un doigt pointé vers', plutôt qu'un doigt qui prétend représenter. La limite, cependant, est que l'intraduisible reste, de facto, artificiellement intraduit. Ou plutôt, si l'on préfère, l'étranger demeure étrange, alors qu'il ne l'est pas, bien souvent, dans la langue et culture source. Le danger, ici, est d'obscurcir ce qui ne l'est pas forcément originellement.

Il devient alors apparent que la traduction n'est pas qu'une question de mot ou qu'une question de sens - pour reprendre la vieille discussion de Saint Jérôme avec Saint Augustin. On pourrait dire que la traduction se place peut-être au-delà du mot et du sens. Qu'elle se place ailleurs. Autrement dit, la traduction est une zone où le sens devient paradoxal : renforcé dans la langue source et fragilisé ou mutilé dans la langue cible. En même temps, cette limite apparente de la langue cible lui permet de s'enrichir de concepts et de notions $a$ priori inexistantes dans sa propre culture, l'obligeant à dépasser ses propres limites. C'est le paradoxe posé par Emily Apter (2005) dans The Translation Zone : «Tout est traduisible, rien n'est traduisible ».

\subsection{L'intraduit}

C'est ici qu'intervient le troisième aspect, souvent délaissé par la théorie de la traduction, qui est celui des 'emprunts', c'est-à-dire des mots, soit non traduits, soit adaptés par notre langue. Nous connaissons tous «weekend», « fjord» et «zen ». Îlots étrangers au cœur de notre langue, ils éclairent des notions qui lui sont (ou lui ont été) radicalement étrangères. Or ces mots sont intéressants car ils participent directement des deux catégories précédemment nommées, l'intraduisible et (de facto) à l'intraductible. D'ailleurs, il y a un mot dano-norvégien 'intraduisible' qui se retrouve sous le feu des projecteurs médiatiques aujourd'hui et qui va nous servir d'exemple : « Hygge ».

Si l'on en croit la définition de Wikipédia, il correspond tout à fait à l'intraduisible : «Ce terme est aujourd'hui principalement utilisé au Danemark et se traduit difficilement dans d'autres langues ». (https://fr.wikipedia.org/wiki/Hygge). Quant à 1' 'intraductible', nous le retrouvons dans la définition, qui le décrit ainsi : «...Le français « cocooning» (sic), « sympa » ne traduisent que partiellement ce concept... ». Si l'équivalence n'est que partielle, cela signifie que le mot tel qu'il existe ne peut trouver une traduction exprimant tous ses sens ou toutes ses valeurs - nous renvoyons de nouveau au travail de Spivak sur la rhétorique.

Ce qui est intéressant, si l'on s'arrête quelques instants sur ce mot, c'est qu'il porte en lui une double caractéristique, toutes les deux liées à la traduction : D'un côté, les Danois euxmêmes en revendiquent l'intraductibilité, qui le rend 'typiquement' local et semble définir une particularité culturelle unique. De l'autre, les Français - et les Anglo-Saxons qui en ont lancé la mode - le mettent en avant précisément pour la même raison, mais inversée, qui est l'Universel. - Si ce mot est 'exotique', il permet toutefois (selon Wikipédia, encore une fois) « de définir un sentiment que nous connaissons tous, mais pour lequel nous n'avons pas de mot» (C'est nous qui soulignons). 
La place de la traduction devient donc ici paradoxale : on utilise ce mot parce qu'on ne peut pas le traduire, même si on comprend ce qu'il représente, sans pouvoir vraiment tout à fait comprendre ce qu'il représente (selon les locuteurs de la langue source). On se rend alors bien compte ici que ces 'emprunts' remplissent un rôle particulier dans la langue, qui est celui qu'on pourrait appeler de 'l'obscurité lumineuse', c'est-à-dire que c'est spécifiquement de par leur condition d'intraduisibilité et d'intraductibilité qu'ils sont inclus dans la langue cible.

Bien sûr, il y aurait aussi, par rapport à ces emprunts, des sous-catégories à établir comme, par exemple, les effets de mode comme «jogging», etc., les mots locaux «Chocolat », qui vient de l'aztèque «Xocolatl», le vocabulaire technique et professionnel comme «Burn-out», «start-up », etc. Ce qui est évident, par contre, c'est que tous ces mots nous obligent à redéfinir constamment notre propre concept de 'traduction' comme phénomène de simple 'passage' ou d'équivalence d'une langue à une autre. L'intraduit nous montre, au contraire, que la 'compréhension', si chère à Saint Jérôme, n'est en fait qu'un des aspects de ce processus, ou plutôt que l'équivalence n'est pas forcément la solution choisie par la langue cible. La coexistence de termes semblables dans la même langue - je pense ici à «weekend » et «fin de semaine»- semblerait indiquer au contraire qu'il se crée une sorte d'égalité de valeur' au travers d'un partage sémantique - «weekend » pour les loisirs, «fin de semaine » pour une indication plus temporelle, par exemple.

L'intraduit se place donc dans le champ lexical comme une fausse transparence ou une fausse opacité, c'est selon, mais en tout cas comme un objet particulier quant à sa nature. Utilisé parce que non-traduisible, il explicite à la fois la limite et la possibilité inclusive de la langue d'accueil, déplaçant dans un même mouvement les frontières entre intraduisible et intraductible, équivalence et domestication, proche et lointain.

Ainsi les éléments intraduits ou adaptés comme « démocratie » ou « destin », doivent être considérés non pas comme des phénomènes en dehors du problème de la traduction, mais comme faisant partie intégralement de cette question, et peut-être comme une des clés possibles pour un renouvellement théorique de ce large champ d'études.

\section{L'implicite}

Une autre notion à laquelle il faut s'atteler en traduction, l'implicite, pose également problème. Le traducteur traduit, on le sait, le sens, les mots, les phrases et les langues, mais il va toujours rester quelque chose d'inconnu ou de caché dans le texte et le discours de l'autre. Comme Lederer $(2003,5)$ le dit « ... chaque échange verbal comporte une partie importante d'implicite. Les phrases, les mots les plus courants sont chargés de sous-entendus. ». Donc, on ne peut traduire avant de comprendre ce quelque chose d'inconnu et de caché. Dans ce contexte, on peut se demander quelles sont les approches que le traducteur doit adapter pour transférer l'implicite ? Après avoir décodé et interprété ce qui reste implicite ou sous-entendu dans le texte, doit-il l'expliciter au lecteur de la langue cible - avec le risque de faire dévier le sens du texte source - ou doit-il plutôt garder l'aspect implicite pour en laisser l'interprétation au lecteur de la langue cible? Si le traducteur ne sait pas décoder l'implicite, la possibilité d'un échec reste évidente. Mais au lieu de parler de l'impossibilité (ou même de l'intraduisibilité, voir ci-dessus), il vaut peut-être mieux parler d'obstacles liés à la traduction de l'implicite.

Il va de soi que la traduction est intimement liée à la langue. En ce qui concerne les obstacles linguistiques, il y a tout un échantillon de phénomènes bien connus : les catégories 
grammaticales, la syntaxe, les typologies (par exemple le français conçu comme une langue exocentrique alors que le danois se caractérise comme une langue endocentrique), l'ordre des mots, les modes et les temps verbaux, à savoir des phénomènes syntaxiques qui sont reliés, d'une manière ou d'une autre, aux aspects culturels et qui les influencent. Regardons p.ex. la position de l'adjectif en français et son importance pour le sens de l'adjectif :

\begin{tabular}{l|l|l} 
& Français & Danois \\
\hline 1a & Une école très catholique & en meget katolsk skole \\
\hline 1b & Une très catholique école & en meget katolsk skole
\end{tabular}

Le locuteur français dispose de plusieurs formulations syntaxiques pour rendre des contenus sémantiques divergents alors que le locuteur danois n'en a pas. En fait, le traducteur se trouve confronté aux obstacles appartenant à des informations de nature culturelle qui, dans cet exemple, se retrouvent dans la position des mots. Une traduction en danois des deux exemples aura un résultat identique mais la traduction dans l'exemple (1b) ne transmet pas toutes les connotations et le savoir culturel dont il est question. En français, c'est la position antéposée de l'adjectif qui renferme des informations implicites, alors que la postposition de l'adjectif ne reflète que des informations dénotatives, neutres. En ce qui concerne les aspects culturels de l'information implicite, l'exemple illustre que les obstacles sont de nature référentielle, ce qui peut également s'illustrer par les divergences appartenant à la culture matérielle et environnementale, par exemple la traduction des institutions politiques et administratives des deux pays: «Le Parlement» qui en France est composé de deux chambres, l'Assemblée Nationale et le Sénat alors que «Folketinget» au Danemark n'est composé que d'une seule chambre.

Il peut s'agir de la culture symbolique ou immatérielle comme par exemple les relations familiales comme «søskende» en danois dont le seul équivalent en français est « frères et sœurs » ou même «mormor » ('grand-mère maternelle'), «farfar » ('grand-père paternel) en danois qui, en français, ne se transmet que par des termes génériques comme « grand-mère » et « grand-père » tout en évitant les informations de parenté maternelle ou paternelle. - Il s'agit donc de phénomènes qui se distinguent par leur référence et les connotations sémantiques et culturelles évoquées. De tels exemples ne reflètent cependant pas de vrais problèmes pour le traducteur qui peut recourir aux explicitations des informations (culturelles).

\subsection{L'ironie - un phénomène de non-explicite}

Par contre, un phénomène comme l'ironie appartient à une autre catégorie qui touche aussi bien la culture et la situation communicationnelle et interpersonnelle que les règles conversationnelles (cf. les maximes de Grice (1976)). L'ironie s'exprime le plus souvent d'une manière non-explicite. Il s'agit d'un phénomène qui se caractérise par l'ambiguïté de l'expression, le non-dit, l'implicite et le sous-entendu. Par conséquent, le décodage et l'interprétation de l'ironie reste un défi particulier, aussi bien pour le récepteur, la cible de l'ironie, que pour le traducteur qui, lui aussi, doit savoir décoder l'implicite - et savoir le transférer dans un autre contexte discursif et culturel. 
L'emploi de l'ironie est toujours intentionnel. Dans la traduction, la tâche du traducteur consiste à repérer les indices, les marqueurs linguistiques et discursifs particuliers s'il y en a - pour ainsi transférer le message implicite et sous-entendu du locuteur. Cependant, les indices restent le plus souvent implicites : il y a peu de formes linguistiques qui sont ironiques en elles-mêmes ${ }^{1}$, mais certains candidats sont susceptibles d'évoquer un effet d'ironie. Il s'agit entre autres d'adjectifs de valeur, de certains mots d'intensification et de particules dialogiques de discours. Ces mots et expressions, appelés déclencheurs d'ironie, n'évoquent cependant pas tout seuls un effet d'ironie, mais dépendent également du co(n)texte, des phénomènes paralinguistiques, du savoir commun et partagé entre le locuteur et le récepteur (la cible), du genre et de la situation conversationnelle et communicative.

Dans les études traductologiques, il existe une certaine réticence quant à la possibilité de transférer l'ironie d'un contexte culturel à un autre sans que se perdent ses effets discursifs et pragmatiques. Dans ce qui va suivre, nous allons illustrer ce défi face auquel se trouve le traducteur à travers quelques exemples relevés dans le roman danois Fasandraberne par Jussi Adler-Olsen et sa traduction en français, Profanation $^{2}$.

Les romans policiers d'Adler-Olsen décrivent un milieu policier et le travail assez rude dans le département Q. Les relations entre le commissaire de police, Carl Mørck et son assistant, Assad, sont empreintes d'un ton jovial, amical - et souvent très ironique. Dans la traduction française, il s'avère que ce ton ironique est parfois absent à cause du choix fait par le traducteur qui se sert souvent d'une stratégie d'explicitation qui va au-delà du discours :

« Det ville nok være mere korrekt, hvis du kaldte dig vicekriminalkommissærassistent eller endnu bedre vicepolitikommissærassistent. Men vil du absolut bruge den titel, så okay for mig. Bare du siger den ekstremt tydeligt, er du med? Og så kan du godt gå ned i

2a køresektionen og gøre vores smukke lig parat. Vi skal til Rørvig. » (Adler-Olsen, 2008, 64)

('gøre vores smukke lig parat' - traduction littérale : 'préparer notre beau cadavre')

« Tu ferais peut-être mieux de te présenter comme assistant d'inspecteur de la PJ ou mieux encore, assistant du vice-commissaire de police. Cela dit, si tu veux continuer à te

2b présenter comme tu l'as fait tout à l'heure, personnellement, je m'en fous. Et maintenant, va préparer la poubelle qui nous sert de voiture. Nous allons à Rørvig. » (Adler-Olsen/ Berg, 2013, 92)

La sémantique de «poubelle » cherche à s'approcher du danois «lig » mais l'aspect implicite, ironique et sous-entendu a disparu dans la traduction choisie à cause de l'explicitation par «qui nous sert de voiture». Le lecteur de la version cible n'a pas à chercher une interprétation de ce qui à l'origine était décrit comme un « cadavre » comme a dû le faire le lecteur de la version source.

\footnotetext{
${ }^{1}$ Sauf les soi-disants polarités d'ironie décrites par Ducrot (2010) qui donne comme exemple un petit saint qui souvent est utilisé pour décrire une personne hypocrite ou l'expression danoise Her går det godt ! ('Ça va bien ici !') utilisée dans une situation qui ne va pas bien du tout.

${ }_{2}$ (Jussi Adler-Olsen. 2008. Fasandraberne. Politikens Forlag / Jussi Adler-Olsen. 2013. Profanation. Albin Michel (trad. par Caroline Berg)
} 
3a du være så venlig at sørge for, at Johan kommer ned til mig om højst ti minutter, Marcus. » (Adler-Olsen, 2008, 99)

«- Tu as raison, c'est une très vieille affaire. Et c'est précisément cette vieille affaire que je vais mettre en avant quand je ferai mon show, comme tu dis, devant la bande de pisse-

3b froid qui vont débarquer du pays du brunost. Alors si tu voulais bien avoir la gentillesse de m'envoyer Johan Jacobsen dans mon bureau dans dix minutes au maximum, je t'en serais très reconnaissant. » (Adler-Olsen/Berg, 2013, 143)

Le fromage de chèvre norvégien «brunost » est couramment utilisé et n'a pas le même ton péjoratif et ironique que «myseost » en danois. Il faut savoir que ce fromage de chèvre est en général très peu apprécié par les Danois. La traduction est donc dépourvue du ton ironique et la description offerte en français devient dénotative et neutre.

«Lav os en kop te, Assad, » sagde Carl nede i kælderen og skubbede Føtex-plasticposerne med Johan Jacobsens materiale hen i hjørnet. «Ikke for meget sukkerstads, okay? » $[\ldots]$

4a «Dejlig myntete med en lille smule sukker, » sagde Assad bagefter og rakte ham en kop, hvor skeen så ud til at kunne stå lodret i sukkerhavet.

Carl tog den, skoldhed og kvalmende, og kværnede den i sig i ét hug. Han var sgu efterhånden ved at vænne sig til mosten. » (Adler-Olsen, 2008, 106-107)

«Fais-nous une tasse de thé, Assad », dit Carl quand ils furent revenus dans leur sous-sol. Il déposa dans un coin du bureau les sacs de supermarché dans lesquels ils avaient entassé tous les éléments que leur avait fournis Johan Jacobsen.

«Pas trop de sucre, hein ? » [...]

4b «Un bon petit thé à la menthe sans trop de sucre», lui annonça Assad un instant plus tard en lui tendant une tasse dans laquelle la cuillère semblait pouvoir tenir à la verticale tant le mélange était sirupeux.

Carl accepta la décoction bouillante et écœurante et l'avala d'un trait. Il commençait à s'habituer à l'étrange breuvage. » (Adler-Olsen/Berg, 2013, 152-153)

Dans cet exemple, toute la situation du thé et du sucre renferme un ton ironique qui repose sur l'antiphrase, ce qui a été bien transfert en français. Le mot de « sukkerstads », qui correspond à peu près à «sucreries » en français, est dans le contexte danois utilisé pour décrire le dégoût que ressent Carl au thé trop sucré fait par son assistant, Assad. La traduction française a perdu ces connotations par l'emploi 'neutre' de « sucre » par la simple description de la trop grande quantité de sucre. Mais encore une fois, le traducteur se sert d'une stratégie d'explicitation «Tant le mélange est sirupeux » et le jeu de mot en danois où on décrit le thé comme « une mer de sucre» disparaît. L'expression «vænne sig til mosten» est une expression dont le sens est ambigü dans la situation en question : soit «il faut s'habituer à une certaine situation » soit, comme dans la traduction actuelle « il faut s'habituer au goût'; donc la traductrice n'a opté que pour une des deux possibilités mais a ainsi perdu le sens 'implicite'. 
Quel que soit le type d'obstacle (linguistique ou culturel) à la traduction, c'est l'implicite qui reste au centre des difficultés face auxquelles se trouve le traducteur. L'implicite peut se définir comme l'information cachée - et offerte - par le texte. Et c'est par la créativité du traducteur que l'implicite peut être rendu dans la traduction, ce qui entraîne inévitablement - toujours quelques déviations de sens dans le texte de la langue cible. Ces déviations ont également affaire avec quelque chose que construit le lecteur/le traducteur à partir du texte en fonction de sa base de connaissance du sujet et du discours en question. La visualisation mentale de la lecture du texte y fait également partie. De plus, le traducteur a souvent un certain souci pour que la traduction soit bien reçue chez le lecteur de la langue cible, ce qui risque de le mener au-delà de la lettre du texte afin d'expliciter, ce qui reste - et qui aurait dû rester (par intention de l'auteur) - implicite et caché dans le texte source.

\subsection{En guise de conclusion}

La traduction représente ainsi une double problématique, d'abord elle se trouve face aux problèmes que pose le passage d'une langue à une autre, à savoir toutes les contraintes stylistiques et linguistiques de l'écriture mais s'y ajoute aussi le respect pour l'écriture de l'auteur. Elle reste partagée entre la soi-disante tradition sourcière et cibliste.

Traduire ne veut pas uniquement dire qu'on traite des aspects explicites de la langue et du discours, ce qui correspondrait à l'équivalence formelle de Nida; il faut également prendre en considération ce qui n'est pas dit explicitement et ce qui reste caché, ce qui, entre autres, est dû à la nature dite synecdochique (voir Lederer 2003). Comme les langues ne sont pas isomorphes, la traduction entre deux langues - et deux cultures - reste intimement liée à cette réalité et y est affectée. Il faut réaliser que cette constatation bouleverse, pour ainsi dire, la conception générale de la traduction, c'est-à-dire l'objet principal de la traduction qui est de traduire les langues, à savoir la forme, mais aussi de considérer le fait que pour transmettre et faire passer la combinaison de l'explicite et de l'implicite, il faut se servir d'une autre combinaison qui semble pertinente dans la langue cible et dont l'expression fonctionne d'une manière naturelle, parfois peut-être même nouvelle. Par conséquent, le traducteur se trouve devant une tâche importante puisqu'il lui est indispensable de savoir transmettre la combinaison de l'explicite et de l'implicite, de les combiner d'une façon appropriée afin de créer un texte et un discours dans la langue cible qui seront perçus comme 'naturels' tout en respectant l'intention implicite, voire cachée, du discours de la langue source.

Le traducteur risque - par accident (peut-être) - de faire émerger l'implicite et de le faire apparaître d'une manière explicite, mais c'est uniquement en cas de nécessité - s'il est impossible de trouver d'autres solutions - que le recours à l'explicitation peut se justifier. Lederer $(2003,11)$ parle de cette problématique en disant :

«Pour transférer les idées, les notions et les émotions de l'original dans leur propre langue, les traducteurs, qu'ils en soient conscients ou non, créent [...] des significations linguistiques explicitement différentes de celles de l'original mais portant un implicite qui, s'il en est tenu compte, permettra aux deux textes de produire un effet équivalent. »

La traduction doit chercher à préserver l'équilibre entre l'explicite et l'implicite autant que possible, alors la tâche du traducteur sera d'affecter le moins possible ce qui reste implicite dans le texte et le discours tout en permettant au lecteur d'en faire sa propre vision mentale dans le respect du texte source. 


\section{Pour conclure}

L'intraduisible et l'implicite sont des phénomènes liminaires, en bordure constante. Ils chevauchent plusieurs territoires allant du culturel à la linguistique. Ils se situent entre les équivalences possibles et impossibles. S'ils ne se déterminent et ne se définissent qu'avec une certaine difficulté, est-ce parce que l'intraduisible et l'implicite équivalent à ce qui est inexeplicable et incompréhensible ? Et l'implicite équivaut-il à une absence de sens qui ne peut se trouver qu'au-delà du texte et du discours ? Ce sont ces questions qu'on a essayé de discuter ci-dessus et pour conclure nous dirons que l'intraduisible - avec ses sous-champs - et l'implicite ne doivent peut-être pas être considérés comme les limites ultimes de la traduction, mais, au contraire, comme leur moteur essentiel, voire central

\section{Bibliographie}

\section{Corpus}

Adler-Olsen, J. 2008. Fasandraberne. Politikens Forlag.

Adler-Olsen, J. 2013. Profanation. Albin Michel (trad. par Caroline Berg).

\section{Reference books}

Apter, E. 2005. The Translation Zone: A New Comparative Literature. Princeton University Press.

Auerbach, E. 1946. Mimesis. The Representation of Reality in Western Literature. Princeton University Press.

Berman, A. 1984. L'épreuve de l'étranger. Culture et traduction dans l'Allemagne romantique. Paris : Gallimard.

Berrendonner, A. 2002. 'Portraits de l'énonciateur en faux naïf'. In Figures du discours et ambiguïté. Semen 15.

Derrida, J. 1985. 'Des tours de Babel'. In J.F. Graham. Difference in Translation. Ithaca, New York: Cornell University Press: 209-248.

Ducrot, O. 1984. 'Ironie et négation'. In Atayan, V. \& Wienen, U. Ironie et un peu plus : hommage à Oswald Ducrot pour son $80^{\text {ème }}$ anniversaire. Berne : Peter Lang : 9-25.

Grice, H.P. 1976. 'Logic and Conversation'. In Cole, P., Morgan, J. Syntax and Semantics, vol 3, Speech Acts. Academic Press : 1-11.

Lederer, M. 2003. 'Le rôle de l'implicite dans la langue et le discours - Les conséquences pour la traduction et l'interprétation. Forum vol. 1, no $1: 1-12$.

Nida, E. 1964/2004. 'Principles of Translation'. In L. Venuti (ed.). The Translation Studies Reader. London \& New York: Routledge : 153-167.

Meschonnic, M. 1999. Poétique du traduire. Lagrasse : Editions Verdier.

Mounin. G. 1963. Les problèmes théoriques de la traduction. Paris: Gallimard.

Sapir, E. 1921. Language. An Introduction to the Study of Speech. New York: Harcourt.

Spivak. G. 1993/2004. 'The Politics of Translation'. In L. Venuti (ed.). The Translation Studies Reader. London \& New York: Routledge: 369-388. 
Venuti, L. 1995. The Translator's Invisibility. London \& New York : Routledge.

Venuti, L. 1998. The Scandals of Translation: Towards an Ethics of Difference. New York : Routledge.

Venuti, L. 2000. The Translation Studies Reader. New York : Routledge. 